where

$$
R_{0}^{2}=x^{2}+y^{2}+c^{2} .
$$

It is evident that if there be a continuous distribution of such nuclei (as enunciated above) over a certain region, we can find the total effect by integration over the region.

\title{
PRACTICAL SOLUTION OF SIMULTANEOUS LINEAR EQUATIONS*
}

By O. L. BOWIE (Watertown Arsenal, Watertown, Mass.)

I. Introduction. The effect of "rounding off" errors in the solution of the set of simultaneous linear equations with constant coefficients

$$
\sum_{i=1}^{n} a_{i j} x_{i}=c_{i} \quad(i=1,2, \cdots, n)
$$

can lead to considerable inaccuracy of solution. Furthermore, the accuracy of an approximate solution of (1) usually cannot be determined from the errors in the individual equations without an excessive amount of additional computation. Unfortunately, most conventional techniques lead unavoidably to "rounding off" errors and the computer is often faced with both of the above problems.

When the form of (1) is such that the well-known" "Classical Iterative" technique can be applied, the difficulties above are usually not encountered. In most practical problems the coefficients and the desired solution can be handled well within the digital capacity of the average desk calculator. Since only the coefficients and approximations of the solution occur in the process, the necessity of "rounding off" is therefore usually eliminated. Accurate estimation of the accuracy of an approximate solution is inherent in the iterative character of the process.

Although rigorous investigations have been made as to the form of (1) sufficient to ensure convergence with the Iterative technique (e.g. footnote 1), the criteria obtained are too impractical to be applied for each case. For all practical purposes, however, a simple rule of thumb is usually sufficient to consider. If (1) can be so ordered that

$$
a_{i j} \gg\left(a_{i 1}, a_{i 2}, \cdots, a_{i, j-1}, a_{i, j+1}, \cdots, a_{i n}\right) \quad j=1,2, \cdots, n,
$$

it can be safely concluded, in general, that the Iterative technique will converge.

It is the aim of this note to demonstrate how conventional techniques can be practicably utilized to transform (1) into an equivalent system which satisfies the conditions (2), thereby enabling the computer to take full advantage of the digital type of solution possible by the Iterative technique. It is the opinion of the author that in general, it is more practical computationally to regard techniques involving "rounding off" errors such as analogue computers, Crout's Method, ${ }^{2}$ etc., as auxiliary tools for obtaining an equivalent system satisfying (2) rather than methods for obtaining a final solution directly.

*Received Dec. 30, 1949.

${ }^{1} \mathrm{H}$. Hotelling, Some new methods of matrix calculation, Ann. Math. Stat. 14, 1-34 (1943).

2P. D. Crout, $A$ short method for evaluating determinants and solving systems of linear equations with real or complex coefficients, Presented at A.I.E.E. Summer Convention, 1941. Published by A.I.E. 1941. 
II. A procedure for the solution of simultaneous linear equations. Let us consider systems of equations (1) which in their original form are such that the Iterative technique leads to a process which diverges or converges too slowly to be practical. Systems of equations (1) which have unique, non-trivial solutions can be represented in matrix form as

$$
A X=C,
$$

where $A$ is the $n \times n$ matrix $\left(a_{i j}\right)$ of rank $n, X$ is the $n \times 1$ column matrix $\left(x_{i}\right)$, and $C$ is the $n \times 1$ column matrix $\left(c_{i}\right)$ where $C \not \equiv 0$. Although a given system of equations (3) will have a unique solution $X$, the converse is not true. In fact, let $Q$ be any $n \times n$ matrix of rank $n$. Then the system of equations

$$
Q A X=Q C
$$

has the same solution as (3) or is "equivalent."

"Diagonalizing" operator matrices, $Q$, can be considered as those operators for which $Q A$ consists of non-zero elements on the principal diagonal alone. Obviously the inverse matrix, $A^{-1}$, belongs to this class since $A^{-1} A=I$, where $I$ is the identity matrix. Little would be gained computationally if, for the solution of a given system, the exact calculation of a diagonalizing operator were required. Such a calculation would be subject to the difficulties arising from "rounding off" errors.

On the other hand, the notion of "equivalent" systems when combined with the Iterative technique lends itself to a practical procedure for the solution of systems of simultaneous linear equations. An approximate diagonalizing operator will in general, be sufficient to determine an equivalent system of such a form that the Iterative technique can be successfully applied. "Pseudo" diagonalizing operators, $Q$, will henceforth be considered as those operator matrices such that $Q A X=Q C$ is in a form such that (2) is satisfied.

Algebraically, the elements $q_{i j}$ of a pseudo diagonalizing operator matrix can be determined as follows: Let $D=\left(d_{i j}\right)$ be an $n \times n$ matrix of rank $n$ such that the elements $d_{i}$ satisfy the type of condition (2). The elements $q_{i j}$ must be determined so that

$$
\left(q_{i j}\right) \cdot\left(a_{i j}\right)=\left(d_{i j}\right) \quad(i, j=1,2, \cdots, n) .
$$

Equation (5) yields the $n$ systems of equations for the successive rows of $Q$,

$$
\sum_{j=1}^{n} a_{i i} q_{k i}=d_{k i} \quad(i=1,2, \cdots, n)
$$

corresponding to $k=1,2, \cdots, n$.

For example, if $d_{i j}$ is chosen as the identity matrix, then the systems (6) represent the algebraic conditions for the elements of the inverse matrix, $A^{-1}$.

III. Practical calculation of pseudo diagonalizing operators. It should be noted first of all that the solution of (6) by most conventional techniques requires little more work than for a single system. This is true because the coefficients in (6) are the same for each of the $n$ systems.

In analogue computers, the successive rows of the matrix $q_{i j}$ are economically obtained by setting up the coefficients $a_{j i}$ once only and then adjusting successively the right hand sides in the order indicated in (6). A practical procedure for the solution of (6) on auto- 
matic calculating devices is obtainable by an obvious extension of "Crout's Method." Since the coefficients in (6) are the same for each system, an obvious extension of "Crout's Method" would be to carry along $(n-1)$ additional columns corresponding to the various right hand sides of (6) in his "auxiliary matrix."

The most practical consideration in the solution of (6) is the degree of freedom the computer has in the choice of $d_{i j}$, the only restriction being that $d_{i j}$ satisfy the type of condition (2). In analogue devices, the computer could adjust iteratively the values of $q_{i j}$ for example, until a suitable set of $d_{i j}$ 's is obtained. Since the original draft of this note was written, this possibility was investigated ${ }^{3}$ and led to very successful results. On the other hand, if techniques designed for the automatic calculator are employed, the most logical approach would seem to choose a particular $d_{i j}$, (for example, the identity matrix) and then examine approximate solutions of (6) so obtained to see if a suitable set of $d_{i}$ 's occur correspondingly.

Since the dominance of the diagonal coefficients indicated in (2) need not be too great in practice, in fact ratios of 10 to 1 are often sufficient to ensure rapid convergence of the Iterative technique, it is evident that quite crude solutions of (6) will often yield pseudo diagonalizing operators. In fact, if the system (1) is reasonably "well-behaved", one can fruitfully consider solutions of approximations of (6). In the typical problem carried out in this note, the coefficients $a_{i j}$ were drastically rounded off in the determination of pseudo diagonalizing operators, yet reasonable values of $q_{i j}$ are obtained.

If the identity matrix, $I$, be chosen for $D$, as was suggested for application of techniques designed for the desk calculator, one might consider a pseudo diagonalizing operator as an approximation of the inverse matrix, $A^{-1}$. It is interesting to note, however, that whereas a good approximation of $A^{-1}$ can be usually considered a satisfactory operator, the converse statement is often far from true.

When several of the equations of a given system are of a form satisfying the conditions (2), calculation can be shortened by carrying these particular equations into the equivalent system directly. To carry the $p$ 'th equation of the original system unaltered in form into the equivalent system, the $p^{\prime}$ th row of the pseudo diagonalizing operator can be immediately written as zero for all elements except that corresponding to the $p$ 'th column, and the element $q_{p p}$ is written as unity.

IV. Pseudo diagonalizing operators from inspection. A technique will now be outlined by which operator matrices for improving the rate of convergence can be determined by inspection.

Consider the systems of equations

$$
R X=S,
$$

where the matrix $R$ can be expressed as the sum of a diagonal matrix of the form $d I$ and a remainder matrix, $\epsilon$, such that $\lim \epsilon_{n \rightarrow \infty}^{n}=0$. In the matrix product $d I, I$ is the identity matrix and $d$ is a scalar greater than or equal to unity. Thus, (7) can be written as

$$
(d I+\epsilon) X=S .
$$

Operator matrices which yield equivalent systems for which the rate of convergence is improved can be written by inspection. As examples,

${ }^{3} \mathrm{~S}$. Ackerman, A low-cost simultaneous equation solver, Watertown Arsenal Report Number WAL $790 / 28$. 


$$
\begin{aligned}
Q_{1}= & (d I-\epsilon)=(2 d I-R) \\
Q_{2}= & \left(d^{2} I-d \epsilon+\epsilon^{2}\right) \\
& \text { etc. }
\end{aligned}
$$

yield the equivalent systems, respectively,

$$
\begin{aligned}
& \left(d^{2} I-\epsilon^{2}\right) X=(d I-\epsilon) S \\
& \left(d^{3} I+\epsilon^{3}\right) X=\left(d^{2} I-d \epsilon+\epsilon^{2}\right) S \\
& \quad \text { etc. }
\end{aligned}
$$

Since $\lim \epsilon_{n \rightarrow \infty}^{n}=0$ is part of the hypothesis, it is evident that, in general, the domination of the diagonal coefficients in (10) will be increased as compared with (8), thus leading to more rapid convergence in the solution of the equivalent system. The operator $Q_{1}$ would seem the most practical for it can often be written down mentally.

V. An Example. The example which is chosen to illustrate the procedures in III and IV is one of the systems used to illustrate "Crout's Method" in footnote 2. Consider the system

$\begin{array}{rrrrrr}x_{1} & x_{2} & x_{3} & x_{4} & =C \\ 12.1719 & 27.3941 & 1.9827 & 7.3757 & =6.6355 \\ 8.1163 & 23.3385 & 9.8397 & 4.9474 & =6.1304 \\ 3.0706 & 13.5434 & 15.5973 & 7.5172 & =4.6921 \\ 3.0581 & 3.1510 & 6.9841 & 13.1984 & =2.5393\end{array}$

The calculation of a pseudo diagonalizing operator $Q$ was made from (6) with $D=I$ (identity matrix). A ten bank Marchant calculator was used in conjunction with the extended "Crout's Method." The coefficients $a_{i j}$ were rounded off to one decimal place and a maximum of three significant figures were kept in the subsequent computations. The pseudo diagonalizing operator thus obtained is

$$
Q=\left(\begin{array}{rrrr}
-1.50 & 2.80 & -2.00 & 0.91 \\
0.56 & -1.00 & 0.75 & -0.37 \\
-0.44 & 0.76 & -0.46 & 0.23 \\
0.44 & -0.81 & 0.53 & -0.17
\end{array}\right)
$$

The corresponding equivalent system, found by multiplying (11) by $Q$ becomes

\begin{tabular}{rrrrrr}
$x_{1}$ & $x_{2}$ & \multicolumn{1}{c}{$x_{3}$} & \multicolumn{1}{c}{$x_{4}$} & & \\
1.109461 & .037260 & -.261959 & -.234686 & $=$ & .138433 \\
-.128583 & .993876 & .384470 & -.062516 & $=$ & .165014 \\
.103639 & .178622 & 1.037369 & .092436 & $=$ & .165157 \\
-.111026 & -.208449 & -.018497 & .978302 & $=$ & .009128
\end{tabular}

The system (13) was solved by the "Classical Iterative" method to nine decimal place accuracy in ten iterations, yielding

$$
\left.\begin{array}{llllllll}
(.15929 & 1130, & .14691 & 7740, & .11257 & 4804, & .06084 & 0731
\end{array}\right)
$$


It is interesting to compare (14) with the solution obtained by Crout. In footnote 2, five significant figures were kept in the computation and the solution of (11) was given as

$$
\text { (0.15942, 0.14687, 0.11261, 0.060806.). }
$$

The equivalent matrices would be in better form of course, if a more careful calculation of (12) were made. Thus, keeping five significant figures in the solution of (6), a pseudo diagonalizing operator was obtained for which the equivalent system was solved to nine decimal accuracy in four iterations.

To illustrate the methods of Section IV, an approximate value of $Q_{1}$ in (9) was chosen to accelerate the solution of (13). To maintain a reasonable number of significant figures, the coefficients of (13) were mentally rounded off to two decimal points. By inspection, therefore, the improving operator, $Q_{1}$, becomes

$$
Q_{1}=\left(\begin{array}{rrrr}
.89 & -.04 & .26 & .23 \\
.13 & 1.01 & -.38 & .06 \\
-.10 & -.18 & .96 & -.09 \\
.11 & .21 & .02 & 1.02
\end{array}\right)
$$

The equivalent system formed by multiplying (13) by (16) required only five iterations to yield (14).

Acknowledgment: The author is indebted to Lyman B. Stewart, Watertown Arsenal, for examination of the computational feasibility of the procedures.

\title{
PLASTIC DESIGN OF BEAMS AND PLANE FRAMES FOR MINIMUM MATERIAL CONSUMPTION*
}

BY JACQUES HEYMAN (Brown University)**

\begin{abstract}
This paper is concerned with the design of plane frames in such a way that the material consumption is a minimum. The method of solution is to set up linear inequalities for the variables involved, and to solve these inequalities by the Dines ${ }^{1}$ method. Three slightly different classes of problem are treated; collapse design under fixed loads, collapse design under varying loads, and shakedown design under varying loads. Illustrative examples of each are given.

1. Introduction. The problem discussed in this paper may be stated as follows: Given the geometrical configuration of a frame which is acted upon by specified loads, how should the various members of the frame be proportioned so that the total material consumption is as small as possible? The type of frame to be considered consists of straight members, all in the same plane. Between joints, the assumption is made that any one member has a uniform cross-section. The moment-curvature curve for any cross-section will be taken to have the general form shown in Fig. 1, where a limit

*Received March 7, 1950. This paper is a shortened version of Technical Report A11-45 prepared for the Office of Naval Research under contract N7onr-358.

**Now Fellow of Peterhouse College, Cambridge, England.

1. Dines, L. L. Systems of linear inequalities. Ann. Math. (2) 20, 191 (1918-1919).
\end{abstract}

